\title{
Comparison of ENDF/B-VII.1 and JEFF-3.2 in VVER-1000 operational data calculation
}

\author{
Jan Frybort ${ }^{\mathrm{a}}$ \\ Czech Technical University in Prague, FNSPE, DNR, V Holesovickach 2, 18000 Prague, Czech Republic
}

\begin{abstract}
Safe operation of a nuclear reactor requires an extensive calculational support. Operational data are determined by full-core calculations during the design phase of a fuel loading. Loading pattern and design of fuel assemblies are adjusted to meet safety requirements and optimize reactor operation. Nodal diffusion code ANDREA is used for this task in case of Czech VVER-1000 reactors. Nuclear data for this diffusion code are prepared regularly by lattice code HELIOS. These calculations are conducted in 2D on fuel assembly level. There is also possibility to calculate these macroscopic data by Monte-Carlo Serpent code. It can make use of alternative evaluated libraries.

All calculations are affected by inherent uncertainties in nuclear data. It is useful to see results of full-core calculations based on two sets of diffusion data obtained by Serpent code calculations with ENDF/B-VII.1 and JEFF-3.2 nuclear data including also decay data library and fission yields data. The comparison is based directly on fuel assembly level macroscopic data and resulting operational data.

This study illustrates effect of evaluated nuclear data library on full-core calculations of a large PWR reactor core. The level of difference which results exclusively from nuclear data selection can help to understand the level of inherent uncertainties of such full-core calculations.
\end{abstract}

\section{Introduction}

The typical sequence of neutronic calculations of nuclear reactors starts with preparation of macroscopic data by detailed lattice calculations on the fuel assembly level. These data are later applied in full-core calculations by deterministic methods.

There are many tools that can be used for the 2D lattice calculations including HELIOS, CASMO, SCALE, and Serpent [1]. The last one employes Monte-Carlo method and allows easy switching of input nuclear data. It uses ACE format [2] for continuous cross-section data and directly evaluated data for decay and fission yields data. It allows demonstration of isolated impact of nuclear data on macroscopic data and full-core calculations.

\section{VVER-1000 nuclear reactor}

It was necessary to select some reactor type for the nuclear data impact comparison. There are two VVER-1000 units [3] in operation in the Czech Republic. These reactors belong in the group of PWR with triangular lattice and hexagonal fuel assemblies. Currently, TVSA-T fuel is used in these reactors. These fuel assemblies are manufactured by TVEL Company [4]. Each fuel assembly, total 163 in the core, contains 312 fuel pins, one central tube (CT) and 18 guide tubes (GT). Schematic drawings of three representatives of fuel assemblies named FTA, FTB, and FTC are in Fig. 1. Due to symmetry of the fuel assemblies only $60^{\circ}$ or $120^{\circ}$ sections are shown.

The fuel assemblies in Fig. 1 contain regular $\mathrm{UO}_{2}$ fuel pins with enrichment levels ranging from $2 \%$ to $4 \%$ show

a e-mail: jan.frybort@fjfi.cvut.cz in green and fuel pins with $\mathrm{Gd}_{2} \mathrm{O}_{3}$ burnable absorber mixed with $\mathrm{UO}_{2}$ shown in red.

\subsection{Serpent and ANDREA calculation model}

Serpent can be seen as an alternative to the established HELIOS code for generation of macroscopic data for ANDREA nodal code. Compared to HELIOS calculations [5], only one average temperatures of all fuel assembly components is assumed for the whole depletion calculation. Also temperature profile along fuel pins radius is not preserved in Serpent and only gadolinium containing fuel pins are radially divided into five regions. These simplifications were introduced to limit Serpent memory requirements and calculation times.

There are multiple branch-off calculations that are identical to HELIOS calculations. They are introduced to provide data for macroscopic data parametrization for fuel or moderator temperatures and other characteristics.

The whole fuel assembly is calculated in Serpent. Symmetry is only used to limit number of unique fuel materials to either 52 or 104. Fuel depletion is calculated individually for all unique fuel pins.

\subsection{Nuclear data}

Two sets of nuclear data are compared: ENDF/B-VII.1 [6] and JEFF-3.2 [7]. These are frequently used evaluated libraries. Cross-section data were processed by NJOY-99 code [8] in form of application libraries in ACE format readable by Serpent. There are total 423 nuclides in ENDF/B-VII.1 library and 472 nuclides in JEFF-3.2 library.

(C) The Authors, published by EDP Sciences. This is an Open Access article distributed under the terms of the Creative Commons Attribution License 4.0 (http://creativecommons.org/licenses/by/4.0/). 


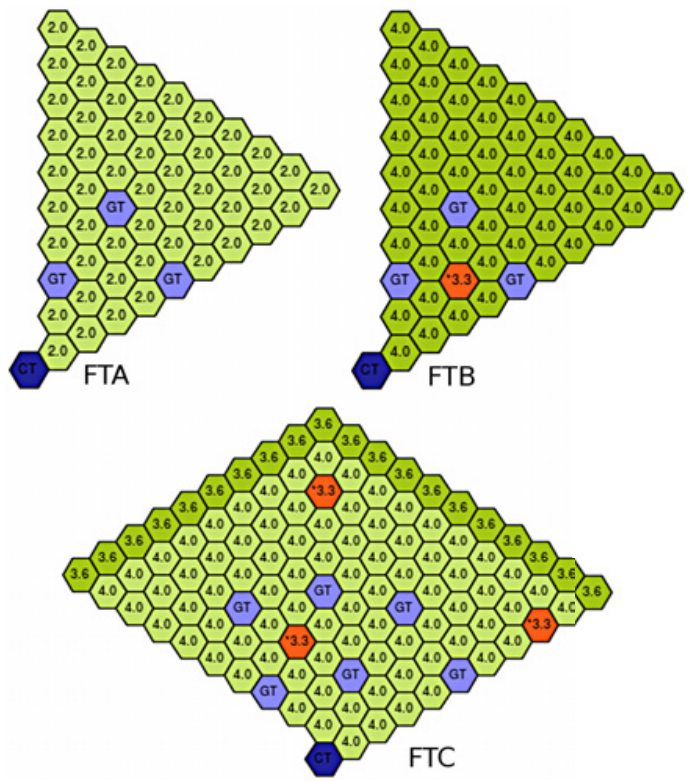

Figure 1. Schematic drawings of fuel assembly types.

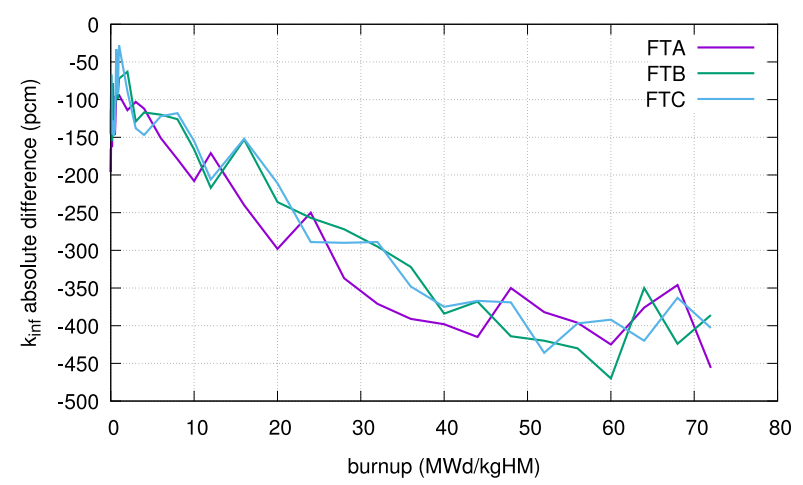

Figure 2. Difference of infinite multiplication number.

Methodology of evaluated data processing by the NJOY-99 code were adopted from JEFF-3.2 validation study [9]. Problems with several nuclides were encountered $-{ }^{63} \mathrm{Cu},{ }^{65} \mathrm{Cu},{ }^{107} \mathrm{Ag},{ }^{109} \mathrm{Ag},{ }^{113} \mathrm{In},{ }^{115} \mathrm{In}$, and ${ }^{157} \mathrm{Gd}$ - that resulted in replacing these data by older versions from JEFF-3.1.2 library. Only the ${ }^{157} \mathrm{Gd}$ data can have potential impact on this analysis, but the only change between the JEFF-3.2 and JEFF-3.1.2 versions is in gamma production spectra in MT102 section of MF6 file.

There was also one replacement in the ENDF/B-VII.1 library, because data for ${ }^{35} \mathrm{Cl}$ are using new formalism for resonances that cannot be interpreted by NJOY-99. This change also has only negligible impact on this analysis.

\section{Macroscopic data}

The first compared parameter of the fuel assemblies is infinite multiplication number. Absolute differences of results using JEFF-3.2 data and ENDF/B-VII.1 data in pcm during the fuel depletion are plotted in Fig. 2. It can be seen that regardless of the fuel type, differences are increasing during fuel depletion.

One of the most important macroscopic parameters of any system is diffusion coefficient. Plot in Fig. 3 shows relative differences of thermal diffusion coefficient in the

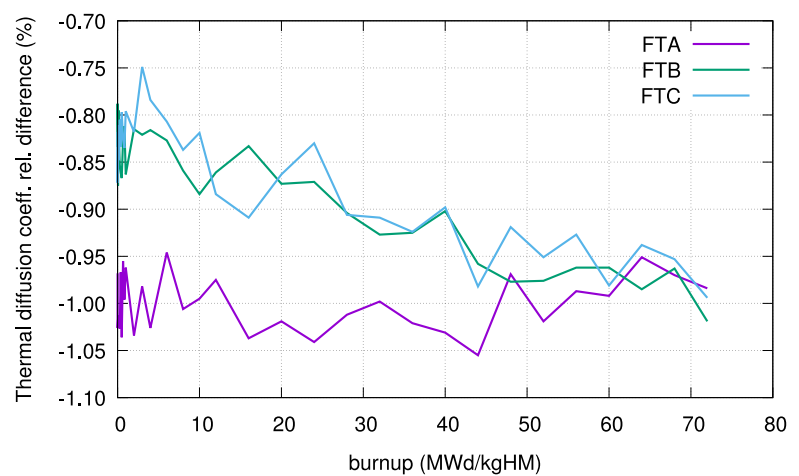

Figure 3. Thermal diffusion coefficient relative difference.

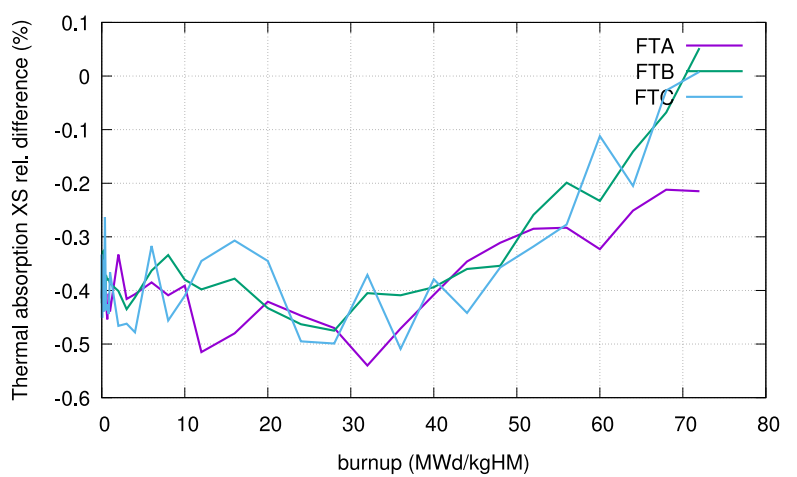

Figure 4. Thermal macroscopic absorption cross-section relative difference.

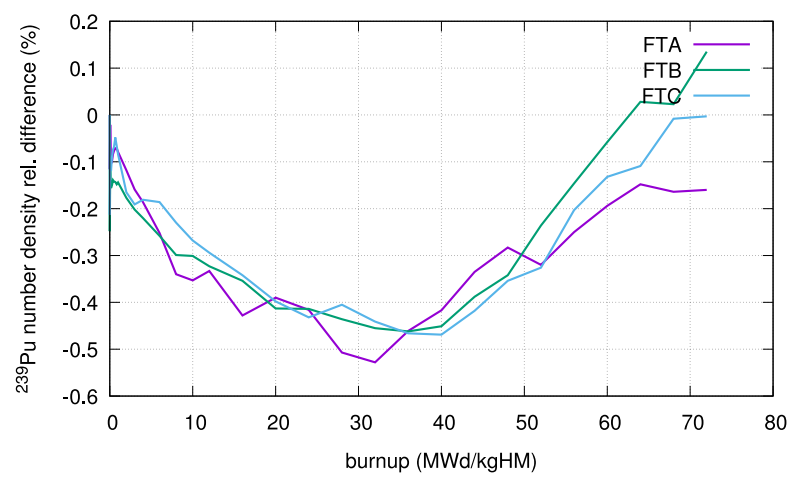

Figure 5. ${ }^{239} \mathrm{Pu}$ number density relative difference.

compared fuel assemblies. All differences are results with JEFF-3.2 data relative to ENDF/B-VII.1 data. Effect of gadolinium presence is clearly visible.

Similar magnitude of differences of macroscopic absorption cross-section but with opposite trend can be seen in plot in Fig. 4. Even for FTA fuel without burnable absorber.

It is also interesting to compare number densities of important nuclides. Two representatives were selected: ${ }^{239} \mathrm{Pu}$ (see Fig. 5) and ${ }^{157} \mathrm{Gd}$ (see Fig. 6). Plutonium is initially accumulated in the system at lower pace with JEFF-3.2 data. Resulting number density is similar for both variants of nuclear data.

Two different behaviours of gadolinium number density time evolution can be seen in plot in Fig. 6 . Differences for burnable absorber containing fuels are low at the beginning of fuel depletion due to self-shielding effect. Final concentration is lower if JEFF-3.2 data are applied, despite that its thermal absorption cross-section 


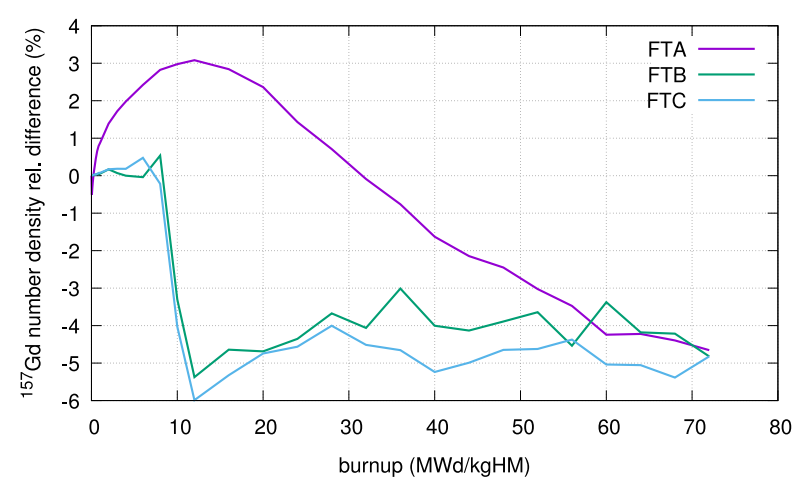

Figure 6. ${ }^{157} \mathrm{Gd}$ number density relative difference.

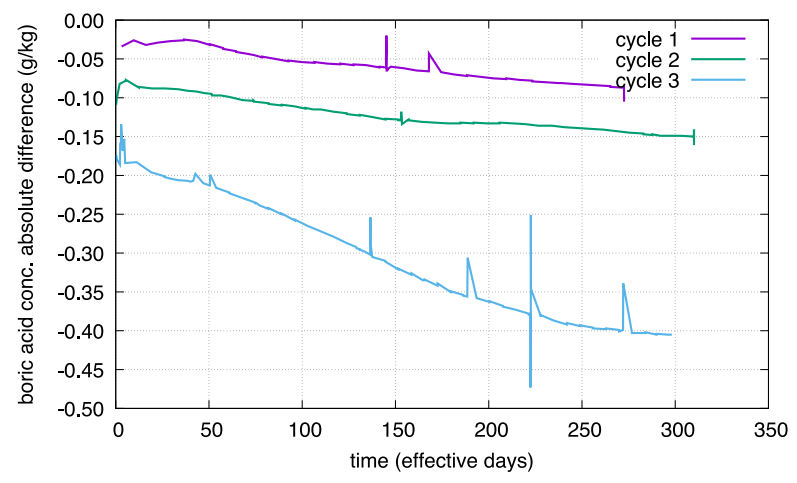

Figure 7. Boric acid concentration absolute difference.

of ${ }^{157} \mathrm{Gd}$ is about $2 \%$ lower compared to ENDF/B-VII.1. It can be explained by about $25 \%$ lower yield of ${ }^{156} \mathrm{Gd}$ by thermal fission of ${ }^{235} \mathrm{U}$ for JEFF-3.2 compared to ENDF/B-VII.1, which results in lower production of ${ }^{157} \mathrm{Gd}$ by subsequent neutron radiative capture.

\section{Full-core data}

The first three cycles of a VVER-1000 unit with TVSA-T fuel were recalculated by ANDREA nodal code with generated macroscopic data. Real operational data (changes of rods positions, power levels, etc.) were used, which is reflected by not smooth time dependence of the analyzed quantities in the following plots.

\subsection{Boric acid, gadolinium, and delayed neutrons}

Boric acid concentration is expressed in $\mathrm{g}$ of $\mathrm{H}_{3} \mathrm{BO}_{3}$ per $\mathrm{kg}$ of water moderator. Figure 7 shows absolute differences of calculated critical boric acid concentrations. Values determined with JEFF-3.2 data are consistently lower and the difference is increasing during the core depletion. It can be explained by differences in ${ }^{135} \mathrm{Xe}$ concentrations. There is about $0.6 \%$ to $0.7 \%$ higher calculated ${ }^{135} \mathrm{Xe}$ concentration with JEFF-3.2 data.

It was shown above in Fig. 6 that depletion and production ${ }^{157} \mathrm{Gd}$ is different with JEFF-3.2 nuclear data. It is also reflected in full-core calculations. Figure 8 shows relative difference of calculated gadolinium concentrations with both data sets. Except for a short period in cycle 3, the JEFF-3.2 data leads to lower calculated gadolinium concentration. This difference can reach up to $4 \%$.

JEFF-3.2 has different structure of delayed neutrons data. They are divided into eight groups instead of typical

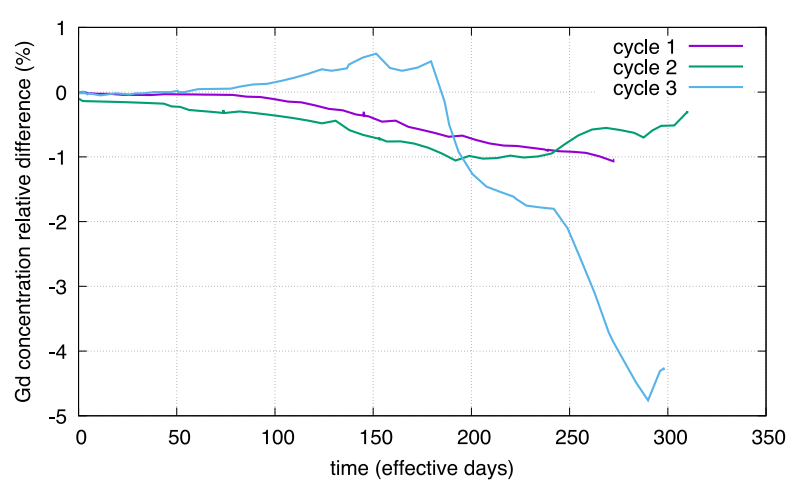

Figure 8. Gadolinium concentration relative difference.

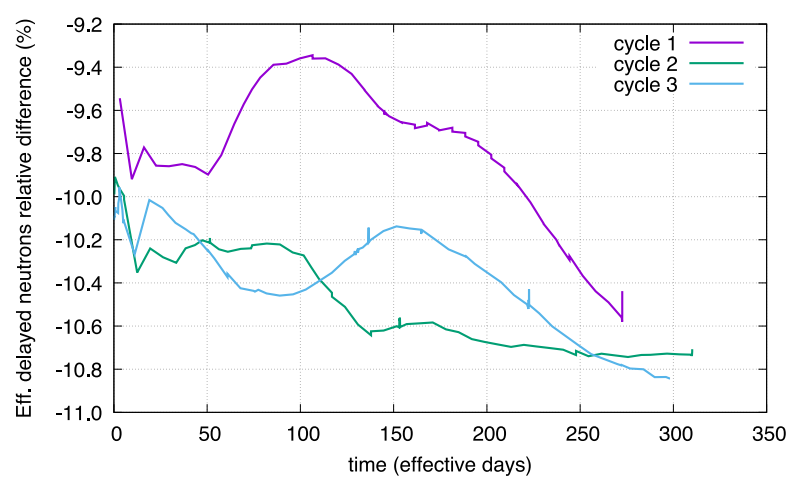

Figure 9. Effective delayed neutrons fraction relative difference.

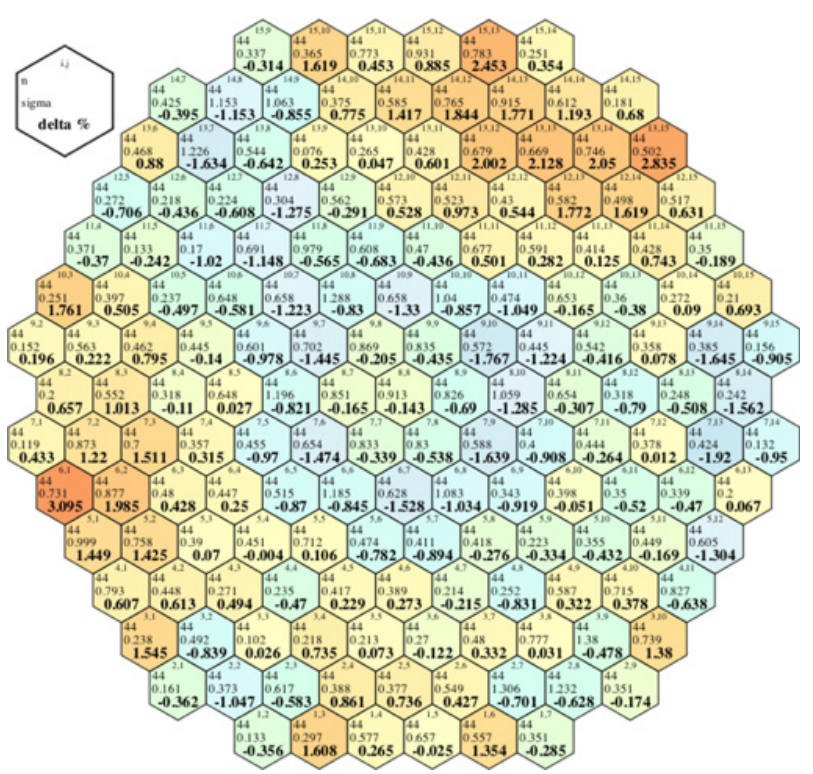

Figure 10. Cycle averaged $\mathrm{C} / \mathrm{E}$ differences of assembly powers with ENDF/B-VII.1 data.

six groups as in ENDF/B-VII.1. As a result, the total delayed neutrons fraction is by about $10 \%$ lower with JEFF-3.2 data, see Fig. 9.

\subsection{Average power distribution}

Results for assembly powers are plotted in Fig. 10 for ENDF/B-VII.1 data and in Fig. 11 for JEFF-3.2 data. These plots are showing relative differences of calculated assembly powers and experimental data from core monitoring. 


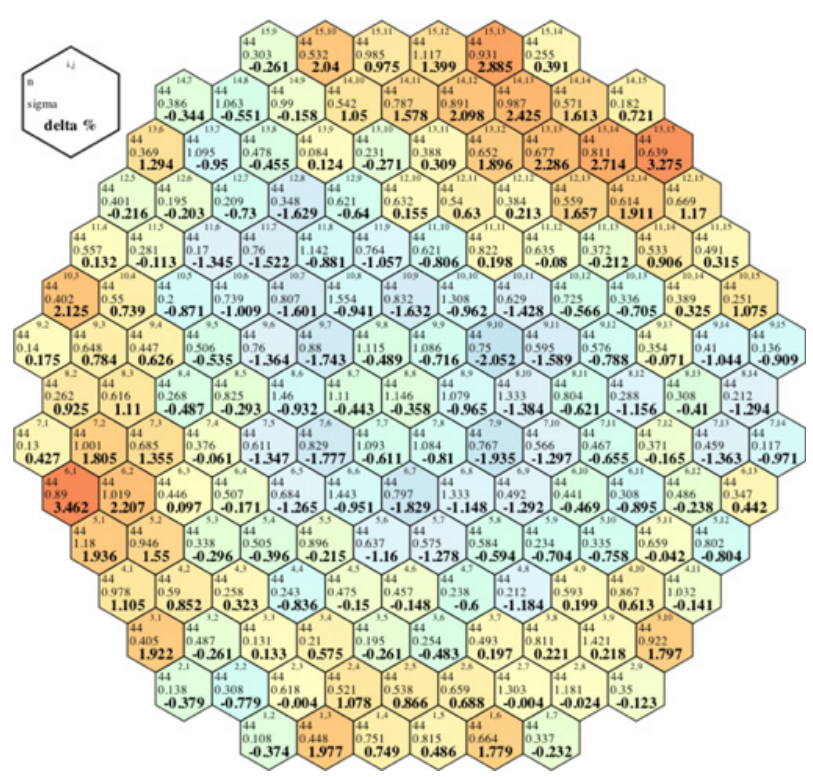

Figure 11. Cycle averaged C/E differences of assembly powers with JEFF-3.2 data.

It can be generally concluded that using JEFF-3.2 data leads to higher power in majority of positions. Differences of relative powers determined by both data sets can reach up to $30 \%$. Better agreement with experimental (operational) data is achieved with ENDF/B-VII.1 data. Only in several positions next to radial reflectors the agreement is better with JEFF-3.2 nuclear data.

\section{Conclusions}

This study analyzed differences in calculated macroscopic and full-core data resulting from switch from ENDF/BVII.1 to JEFF-3.2 nuclear data. The switch included crosssection data, decay and fission yield data.

It was found that the calculated infinite multiplication is lower for JEFF-3.2. The difference is reaching hundreds of pcm. Also thermal diffusion coefficient is smaller by about $1 \%$. Lower difference was observed for thermal macroscopic absorption cross-section. Different nuclear data resulted also in change of ${ }^{239} \mathrm{Pu}$ and ${ }^{157} \mathrm{Gd}$ production and depletion.

Based on the full-core data it can be seen that difference of several tenths of $\mathrm{g} / \mathrm{kg}$ of boric acid between calculations and measurements can be attributed to nuclear data. Important is also difference in delayed neutrons data that is key for reactor kinetics.

This study contributed to understanding of inherent uncertainty in nuclear data that cannot be easily eliminated and that limits precision of full-core calculations.

This study was supported by Technology Agency of the Czech Republic grant TA04021349.

\section{References}

[1] Leppänen J., Viitanen T., Valtavirta V. and Kaltiaisenaho T., Ann. Nucl. Energy 82, 142-150 (2015)

[2] Robert C. Little, V\&V of MCNP and Data Libraries at Los Alamos (LANL, Los Alamos, 2012) 26 p.

[3] International Atomic Energy Agency, In-Core Fuel Management Code Package Validation for WWERs (IAEA, Vienna, 1995) $156 \mathrm{p}$.

[4] TVEL JSC, Nuclear Fuel for VVER Reactors (TVEL JSC, Moscow, 2011) $12 \mathrm{p}$.

[5] Heraltova L., Frybort J., ANDREA recalculation of operation history of VVER-440 (BUT, Brno, 2014) 5 p.

[6] Chadwick M.B., Herman M., Oblozinsky P., et al., Nuclear Data Sheets 12, 2887-2996 (2011)

[7] Santamarina A., Bernard D., Rugama Y., The JEFF3.1.1 Nuclear Data Library (OECD NEA, Paris, 2009) $62 \mathrm{p}$.

[8] MacFarlane R.E., NJOY99.0: Code System for Producing, Pointwise and Multigroup Neutron and Photon Cross Sections from ENDFB Data (LANL, Los Alamos, 1999) $810 \mathrm{p}$.

[9] Cabellos O., Processing of the new JEFF-3.2 T4 Cross Section Library with the NJOY Code System into Various Formats for Testing Purposes (OECD NEA, Paris, 2012) 48 p. 